\title{
AN UNUSUAL MOVING BOUNDARY CONDITION ARISING IN ANOMALOUS DIFFUSION PROBLEMS*
}

\author{
D. A. EDWARDS ${ }^{\dagger}$ AND D. S. COHEN $\ddagger$
}

\begin{abstract}
In the context of analyzing a new model for nonlinear diffusion in polymers, an unusual condition appears at the moving interface between the glassy and rubbery phases of the polymer. This condition, which arises from the inclusion of a viscoelastic memory term in our equations, has received very little attention in the mathematical literature. Due to the unusual form of the moving-boundary condition, further study is needed as to the existence and uniqueness of solutions satisfying such a condition. The moving boundary condition which results is not solvable by similarity solutions, but can be solved by integral equation techniques. A solution process is outlined to illustrate the unusual nature of the condition; the profiles which result are characteristic of a dissolving polymer.
\end{abstract}

Key words. integral equations, moving boundary-value problems, non-Fickian diffusion, polymer-penetrant systems

AMS subject classifications. 35B25, 35C15, 35C20, 35K60, 35R35, 73F15, 76R99, 80A22

1. Introduction. In recent years, engineers and scientists have found a panoply of uses for polymers and other synthetic materials. These new materials promise to revolutionize entire industries and create new ones. The sudden explosion in the development of these materials has thrust materials science to the forefront of mathematical applications, especially since there is so little mathematical modeling of the dynamics of synthetic materials. Mathematicians are also handicapped by the debate raging among chemical engineers and materials scientists as to the exact physical mechanisms involved. However, all agree that the unusual behavior exhibited by these new materials indicates that the standard Fickian flux $\mathbf{J}=-D(C) \nabla C$, where $D(C)$ is the second-order diffusion tensor and $C$ is the concentration, is not general enough to model the desired behavior accurately. It is also a growing consensus that some sort of viscoelastic stress plays a major role in diffusion in many of these materials, sharing dominance with molecular diffusion.

The promise that these new types of materials hold is astounding. New types of adhesives will adhere more while weighing less [1], [2]. "Smart" polymer gels will forever change how doctors administer medicine, as they abandon standard global delivery methods in favor of internal or external on-site administrations [3]-[5]. Microlithographic patterning using polymer substrates has emerged as a major technology [6]. Polymer films have great value in protective clothing, equipment, or sealants $[7]$.

*Received by the editors August 18, 1993; accepted for publication May 10, 1994. This work was performed under United States Army Research Office (Durham) contract DAAL03-89-K-0014, National Science Foundation grant DMS-9024963, Air Force Office of Scientific Research grant AFOSR91-0045, and Department of Energy grant W-7405-ENG-36 at the Center for Nonlinear Studies at Los Alamos. Additional support was provided by a National Science Foundation Graduate Fellowship.

$\dagger$ Applied Mathematics, California Institute of Technology, Pasadena, California 91125. Mailing address, Courant Institute of Mathematical Sciences, New York University, New York, New York 10012-1185 (edwardsd@cims.nyu.edu).

$¥$ Applied Mathematics, California Institute of Technology, Pasadena, California 91125 (dsc@ama. caltech.edu). 
Polymer-penetrant systems are particularly interesting since much of the observed behavior is inconsistent with a purely Fickian diffusion model. In particular, unless pathological conditions are met, the moving Fickian front always proceeds with speed proportional to $t^{-1 / 2}$. However, in so-called case II diffusion in polymers, concentration fronts propagate with constant speed [3], [8]. These fronts are usually sharp, and often the concentration flux into the phase change boundary is less than the concentration flux out! All of these characteristics are inconsistent with those of the Fickian diffusion model. Though the concentration fronts are sharp, there is no discontinuity in $C$, as observed in other, more standard chemical systems [9].

The type of polymers which we wish to study can occupy one of two phases: glassy or rubbery. In the glassy state, the polymer has a finite relaxation time associated with the length of the polymer in relation to the entanglement network. This nonlocal effect implies that there will be a stress associated with the "memory" of the polymer with respect to its concentration history. In the rubbery state, the polymer swells, making the relaxation time almost instantaneous. Hence, the "memory" of the polymer in the rubbery state is very faint. In addition, in many, but not all cases, there is a great increase in the diffusion coefficient as the polymer changes from the glassy to rubbery state.

In order to incorporate this more complicated behavior into the flux, we propose the following much more general model for the flux:

$$
\mathbf{J}=-\sum_{n=1}^{\infty} D_{n}(C) \nabla \int_{\Omega} \int_{-\infty}^{t} \mathcal{F}_{n}\left[C\left(\mathbf{x}^{\prime}, t^{\prime}\right)\right] \mathcal{G}_{n}\left[\mathbf{x}-\mathbf{x}^{\prime}, t-t^{\prime}, C\left(\mathbf{x}^{\prime}, t^{\prime}\right)\right] d t^{\prime} d \mathbf{x}^{\prime}
$$

where the $D_{n}$ are second-order tensors, the $\mathcal{F}_{n}$ are general differential operators on $C$ which model the dependency of $\mathbf{J}$ on different dynamical processes, and the $\mathcal{G}_{n}$ are general nonlinear hereditary kernels. Each term in the expansion represents a flux contribution from a different source, such as molecular diffusion or viscoelastic effects. This form for the flux is general enough to model accurately many more types of anomalous diffusive behavior than simply those associated with polymer-penetrant systems. Furthermore, note that if we let $\mathcal{F}_{n}=\delta_{1 n} C\left(\mathbf{x}^{\prime}, t^{\prime}\right)$ and $\mathcal{G}_{n}=\delta_{1 n} \delta\left(\mathbf{x}-\mathbf{x}^{\prime}, t-t^{\prime}\right)$ we obtain the Fickian diffusion flux.

In [10]--[16] Cohen and his colleagues have specialized equation (1.1) to several different cases of viscoeleastic diffusion. The main purpose of this paper is to formulate and discuss the unusual moving boundary conditions which arise when one uses the flux in (1.1) to solve the standard diffusion equation $C_{t}=-\nabla \cdot \mathbf{J}$. In the next section we will specialize $\mathcal{F}_{n}$ and $\mathcal{G}_{n}$ to the particular case of viscoelastic stress we wish to consider and consider the extra complication of dynamics at a moving boundary.

2. Governing equations. Consider a domain $\Omega$ which is divided into two disjoint connected subdomains $\Omega_{1}$ and $\Omega_{2} . \Omega_{1}$ is the region in which the polymer is in the glassy state, while $\Omega_{2}$ is the region in which the polymer is in the rubbery state. We specify the value of the concentration in the interior of $\Omega$ at time $t=0$ and on the boundary $\partial \Omega$ for all time. We could just have easily specified the flux on the boundary, though in the systems we wish to study the concentration is usually specified. In addition, the standard diffusion equation holds for the concentration in both domains, though the flux $\mathbf{J}$ may be different in each region. Specifically, we are considering the following system of equations:

$$
C_{t}=-\nabla \cdot \mathbf{J}_{1}, \quad \mathbf{x} \in \Omega_{1} ; \quad C_{t}=-\nabla \cdot \mathbf{J}_{2}, \quad \mathbf{x} \in \Omega_{2},
$$




$$
C(\mathbf{x}, t)=C_{b}(t), \quad \mathbf{x} \in \partial \Omega ; \quad C(\mathbf{x}, 0)=C_{i}(\mathbf{x}), \quad \mathbf{x} \in \Omega
$$

In polymer-penetrant systems, there is no discontinuity of concentration at the front; it is merely very sharp. The front is characterized by a transition value $C=C_{*}$ at which the glass-rubber phase transition takes place. Hence, we specify the following condition:

$$
C=C_{*}, \quad C_{t}>0, \quad \mathbf{x}=\mathbf{s}(t) .
$$

We include the derivative in (2.2) since we mathematically define the glassy region as the region where $C \leq C_{*}$ and the rubbery region as the region where $C>C_{*}$.

At the moving boundary $\mathbf{s}(t)$ between the two regions a phase change takes place. While the same physical mechanisms do not govern here, it is instructive to recall the boundary condition used in the classical Stefan problem [9], where a change of phase takes place between ice and water. In that problem, the following condition holds:

$$
\mathbf{J}_{2}(\mathbf{s}(t), t) \cdot \mathbf{n}-\mathbf{J}_{1}(\mathbf{s}(t), t) \cdot \mathbf{n} \equiv-[\mathbf{J} \cdot \mathbf{n}]_{\mathbf{s}}=a \dot{\mathbf{s}} \cdot \mathbf{n}, \quad \mathbf{x} \in \mathbf{s}(t)
$$

Here $a$ is the phase change parameter. Equation (2.3) states that the difference between the flux into and out of the front is used up in the phase transition. In a standard Stefan problem, the parameter $a$ has a physical interpretation (namely the latent heat) and is always positive. We will see that in polymer-penetrant systems the interpretation of $a$ is not so straightforward; in later sections we will outline the solution of equations where $a$ is negative. Like the latent heat in a Stefan problem, $a$ must be known in order to solve the problem. However, there are experiments which can be performed to determine $a$ just as there are experiments which can be performed to determine the latent heat of a substance. Such an experiment is outlined in $\S 5$.

Experimentalists note several important properties in the polymer-penetrant systems which we are trying to study. First, there is a finite relaxation time [17] when the polymer is in the glassy state. This indicates the presence of a viscoelastic memory term in our flux. The polymer is affected by past values of the concentration and its time derivative [8], [18], [19], so we make the following definitions:

$$
\mathcal{F}_{1}=C, \quad \mathcal{G}_{1}=\delta\left(\mathbf{x}-\mathbf{x}^{\prime}, t-t^{\prime}\right), \quad \mathcal{F}_{2}=H\left(t^{\prime}\right) f\left(C, C_{t}\right), \quad \mathcal{F}_{n}=0, \quad n>2
$$

$$
\mathcal{G}_{2}=\exp \left[-\int_{t^{\prime}}^{t} \beta(C(\mathbf{x}, z)) d z\right] d t^{\prime}, \quad D_{1}(C)=D(C), \quad D_{2}(C)=E(C) .
$$

Here $H\left(t^{\prime}\right)$ is the Heaviside step function, $f$ is some general scalar function, $\beta(C)$ is the inverse of the relaxation time for the polymer, and $E(C)$ is a tensor. Specific forms for $f, \beta$, and $E$ will be chosen later. Hence we may write the flux as

$$
\mathbf{J}=-D(C) \nabla C-E(C) \nabla \sigma, \quad \text { where }
$$

$$
\sigma=\int_{0}^{t}\left[f\left(C\left(\mathbf{x}, t^{\prime}\right), C_{t}\left(\mathbf{x}, t^{\prime}\right)\right)\right] \exp \left[-\int_{t^{\prime}}^{t} \beta(C(\mathbf{x}, z)) d z\right] d t^{\prime}
$$


We also note that $\sigma$ plays the role of the viscoelastic memory term, and that the definition of $\sigma$ in $(2.5 \mathrm{~b})$ implies that

$$
\sigma(\mathbf{x}, 0)=0
$$

Lastly, we need a condition for the stress at the front. We follow the work of Knauss and Kenner [20], where the derivative of stress with respect to a state variable has a jump in slope at the phase transition, but the actual stress is continuous:

$$
\sigma\left(\mathbf{s}^{-}(t), t\right)=\sigma\left(\mathbf{s}^{+}(t), t\right)
$$

Using equations (2.5) in (2.1a) and making $\sigma$ another dependent variable, we have the following system of partial differential equations:

$$
\begin{gathered}
C_{t}=\nabla \cdot(D(C) \nabla C+E(C) \nabla \sigma) \\
\sigma_{t}+\beta(C) \sigma=f\left(C, C_{t}\right) .
\end{gathered}
$$

Here we have combined the two equations on either side of the front. We will allow discontinuities in $\beta(C), D(C), E(C)$, and $f$ across the front, indicating distinct values in the glassy and rubbery regions. Note that equation $(2.8 \mathrm{~b})$ is reminiscent of the memory integral term in viscoelasticity theory.

Now using equation (2.5a) in our flux condition (2.3), we have the following:

$$
\left[D\left(C_{*}\right) \nabla C+E\left(C_{*}\right) \nabla \sigma\right]_{\mathbf{s}} \cdot \mathbf{n}=a \dot{\mathbf{s}} \cdot \mathbf{n} .
$$

This is the condition at the moving boundary which replaces the standard Stefan flux condition; it is clearly more complicated than the standard Stefan condition, and the interesting details of these complications will be explored in the next section.

3. A one-dimensional problem with flux condition. For analytical tractability, we first consider a one-dimensional problem on a semi-infinite domain, in which case $(2.8),(2.9),(2.1 \mathrm{~b}),(2.6),(2.2)$, and (2.7) become

$$
\begin{gathered}
C_{t}=\left(D(C) C_{x}+E(C) \sigma_{x}\right)_{x}, \quad x>0 \\
\sigma_{t}+\beta(C) \sigma=f\left(C, C_{t}\right), \quad t>0, \\
{\left[D\left(C_{*}\right) C_{x}+E\left(C_{*}\right) \sigma_{x}\right]=a \dot{s},} \\
C(x, 0)=C_{i}(x), \\
C(0, t)=C_{b}(t), \quad C_{b}^{\prime}(t)>0 \quad \forall t>0, \quad C_{b}(t)>C_{*} \text { for some } t>0, \\
\sigma(x, 0)=0,
\end{gathered}
$$




$$
\begin{gathered}
C(s(t), t)=C_{*}, \quad C_{t}(s(t), t)>0 \\
\sigma^{r}(s(t), t)=\sigma^{g}(s(t), t)
\end{gathered}
$$

We take $E(C)$ and $D(C)$ to be scalar nonnegative strictly increasing functions of $C$, reflecting the situation typically encountered in controlled-release pharmaceuticals [3]-[5]. Note that in one dimension $\sigma$ can be interpreted as being analogous to stress. We also label the glassy region (the region ahead of the front) with a superscript $g$ and the rubbery region (the region behind the front) with a superscript $r$.

Since we expect our front $x=s(t)$ to be monotonically increasing in $t$, we may invert to write the front as $t=\bar{s}(x)$. We then solve equation (3.1b) subject to (3.4) and $(3.5 \mathrm{~b})$ to yield

$$
\sigma^{g}(x, t)=\int_{0}^{t}\left[f\left(C\left(x, t^{\prime}\right), C_{t}\left(x, t^{\prime}\right)\right)\right] \exp \left[-\int_{t^{\prime}}^{t} \beta(C(x, z)) d z\right] d t^{\prime}
$$

$$
\begin{array}{r}
\sigma^{r}(x, t)=\int_{0}^{\bar{s}}\left[f\left(C\left(x, t^{\prime}\right), C_{t}\left(x, t^{\prime}\right)\right)\right] \exp \left[-\int_{t^{\prime}}^{\bar{s}} \beta(C(x, z)) d z-\int_{\bar{s}}^{t} \beta(C(x, z)) d z\right] d t^{\prime} \\
+\int_{\bar{s}}^{t}\left[f\left(C\left(x, t^{\prime}\right), C_{t}\left(x, t^{\prime}\right)\right)\right] \exp \left[-\int_{t^{\prime}}^{t} \beta(C(x, z)) d z\right] d t^{\prime}
\end{array}
$$

We have expanded the argument of the exponential in the first term of $\sigma^{r}$ since we expect the relaxation time to undergo a discontinuous jump at $C=C_{*}$, in agreement with experiments [17].

In general, if we have functions

$$
\begin{aligned}
& f^{g}(x, t)=\int_{0}^{t} f^{(1)}(x, z ; t) d z \\
& f^{r}(x, t)=\int_{0}^{\bar{s}(x)} f^{(3)}(x, z ; t) d z+\int_{\bar{s}(x)}^{t} f^{(2)}(x, z ; t) d z
\end{aligned}
$$

then Leibniz's rule for differentiation states that

$$
\left[f_{x}\right]_{\bar{s}}=\int_{0}^{\bar{s}}\left[f^{(1)}-f^{(3)}\right]_{x}(x, z ; \bar{s}) d z+\bar{s}^{\prime}\left[f^{(2)}(x, \bar{s} ; \bar{s})-f^{(3)}(x, \bar{s} ; \bar{s})\right] .
$$

Note that since we have changed variables, $\left[f_{x}\right]_{\bar{s}}=f_{x}^{g}\left(x, \bar{s}^{-}(x)\right)-f_{x}^{r}\left(x, \bar{s}^{+}(x)\right)$.

Now using equations (3.6) in (3.7), we have the following:

$$
\begin{aligned}
{\left[E\left(C_{*}\right) \sigma_{x}\right]_{\bar{s}}=\int_{0}^{\bar{s}}\left\{\mathcal{J}[C]+\bar{s}^{\prime} f\left(C, C_{t}\right)\left[E\left(C_{*}\right) \beta\left(C_{*}\right)\right]_{\bar{s}}\right\} \exp [} & \left.-\int_{t^{\prime}}^{\bar{s}} \beta(C(x, z)) d z\right] d t^{\prime} \\
& -E\left(C_{*}^{+}\right) \bar{s}^{\prime}\left[f\left(C_{*}, C_{t}\right)\right]_{\bar{s}}
\end{aligned}
$$

where

$$
\mathcal{J}[C]=\left[E\left(C_{*}\right)\right]_{\bar{s}}\left[f_{x}\left(C\left(x, t^{\prime}\right), C_{t}\left(x, t^{\prime}\right)\right)-f\left(C, C_{t}\right) \int_{t^{\prime}}^{\bar{s}} \beta^{\prime}(C(x, z)) C_{x}(x, z) d z\right] .
$$


Simplifying and transforming to our original variables, we have

$$
\begin{aligned}
{\left[E\left(C_{*}\right) \sigma_{x}\right]_{\bar{s}}=\int_{0}^{\bar{s}} \mathcal{J}[C] \exp \left[-\int_{t^{\prime}}^{\bar{s}} \beta(C(x, z)) d z\right] d t^{\prime} } \\
\quad+\frac{\left[E\left(C_{*}\right) \beta\left(C_{*}\right)\right]_{s} \sigma(s(t), t)}{\dot{s}}-\frac{E\left(C_{*}^{+}\right)\left[f\left(C_{*}, C_{t}\right)\right]_{s}}{\dot{s}}
\end{aligned}
$$

which makes our flux condition (3.2)

$$
\begin{aligned}
{\left[D\left(C_{*}\right) C_{x}\right]_{s}+\int_{0}^{\bar{s}} \mathcal{J}[C] \exp } & {\left[-\int_{t^{\prime}}^{\bar{s}} \beta(C(x, z)) d z\right] d t^{\prime} } \\
+ & \frac{\left[E\left(C_{*}\right) \beta\left(C_{*}\right)\right]_{s} \sigma(s(t), t)}{\dot{s}}-\frac{E\left(C_{*}^{+}\right)\left[f\left(C_{*}, C_{t}\right)\right]_{s}}{\dot{s}}=a \dot{s} .
\end{aligned}
$$

There are several interesting things to note in equation (3.10). First, it may seem that we have not simplified matters much, since $\sigma$ still appears in our flux condition. However, in practice it is much easier, both analytically and numerically, to determine $\sigma$ than $\sigma_{x}$. Note also that we have a negative contribution to the left-hand side, so we cannot be assured that $a$ is positive, as was always true in the latent heat formulation.

More interesting is the appearance of $\dot{s}(t)$ in the denominator of some of our flux terms. This condition is highly unusual and leads to nonstandard front motion, especially when one considers the fact that $\dot{s}(t)$ may also appear in the expressions for the concentration and the flux. For instance, it is clear from (3.10) that if all the flux and stress terms are bounded as $t \rightarrow \infty, \dot{s}(t)$ must approach a constant as $t \rightarrow \infty$. This means that these diffusive systems will have fronts which move with constant speed. Such fronts are characteristic of polymer-penetrant systems.

In general, the behavior induced by (3.10) is highly complicated. Boundary conditions of the type of (3.10) are unusual in the mathematical literature, and certainly no comprehensive theoretical study of such a condition has ever been attempted. Hopefully such a study will be made in light of the fact that this paper will demonstrate that such an odd condition as (3.10) does occur in real-world problems. In the next section we will specialize our problem further, thereby making it possible to find analytical solutions.

4. Further simplifications: a tractable problem. The first simplification we choose to make is that in both regions $f$ takes the same simple form:

$$
f\left(C, C_{t}\right)=\mu C+\nu C_{t}
$$

Here $\mu$ and $\nu$ are positive constants. We choose this form because it is simple to analyze and accurately captures the dominant physical processes in the system [10].

Then equations (3.1b) and (3.10) become

$$
\sigma_{t}+\beta(C) \sigma=\mu C+\nu C_{t}
$$

$$
\begin{aligned}
{\left[D\left(C_{*}\right) C_{x}\right]+\int_{0}^{\bar{s}} \mathcal{J}[C] \exp } & {\left[-\int_{t^{\prime}}^{\bar{s}} \beta(C(x, z)) d z\right] d t^{\prime} } \\
+ & \frac{\left[E\left(C_{*}\right) \beta\left(C_{*}\right)\right]_{s} \sigma(s(t), t)}{\dot{s}}-\frac{E\left(C_{*}^{+}\right)\left[\mu C_{*}+\nu C_{t}\right]_{s}}{\dot{s}}=a \dot{s} .
\end{aligned}
$$


Now using the first of equations (3.5a) and its total derivative with respect to $t$, we have

$$
\begin{aligned}
{\left[\left(D\left(C_{*}\right)+\nu E\left(C_{*}^{+}\right)\right) C_{x}\right]_{s}+\int_{0}^{\bar{s}} \mathcal{J}[C] \exp \left[-\int_{t^{\prime}}^{\bar{s}} \beta(C(x, z)) d z\right] d t^{\prime} } & \\
+\frac{\left[E\left(C_{*}\right) \beta\left(C_{*}\right)\right]_{s} \sigma(s(t), t)}{\dot{s}} & =a \dot{s} .
\end{aligned}
$$

Hence we see that this particular form for $f$ dictates a simple relationship between the viscoelastic flux contribution and the concentration flux contribution.

Experiments have shown that variations in the relaxation time within phases seem to contribute little to the overall behavior. Therefore, we average the relaxation time in each phase and use its overall value there. Thus we have

$$
\beta(C)= \begin{cases}\beta_{g}, & 0 \leq C \leq C_{*}, \\ \beta_{r}, & C>C_{*} .\end{cases}
$$

Under these assumptions, our flux condition (4.4) becomes

$$
\begin{array}{r}
{\left[\left(D\left(C_{*}\right)+\nu E\left(C_{*}^{+}\right)\right) C_{x}\right]_{s}+\int_{0}^{\bar{s}}\left[E\left(C_{*}\right)\right]_{s}\left[f_{x}\left(C\left(x, t^{\prime}\right), C_{t}\left(x, t^{\prime}\right)\right)\right] e^{-\beta_{g}\left(\bar{s}-t^{\prime}\right)} d t^{\prime}} \\
+\frac{\left[E\left(C_{*}\right) \beta\left(C_{*}\right)\right]_{s} \sigma(s(t), t)}{\dot{s}}=a \dot{s} .
\end{array}
$$

We note that changes in $E(C)$ also do not contribute significantly to the behavior of the system. Hence, we approximate $E(C)$ by its average value in the entire polymer, which we denote by $E$, a positive constant. Doing so, (3.1a) and (4.6) become the following:

$$
\begin{gathered}
C_{t}=\left(D(C) C_{x}\right)_{x}+E \sigma_{x x} \\
{\left[\left(D\left(C_{*}\right)+\nu E\right) C_{x}\right]_{s}+\frac{E\left(\beta_{g}-\beta_{r}\right) \sigma(s(t), t)}{\dot{s}}=a \dot{s}}
\end{gathered}
$$

Note that since $\beta_{r}>\beta_{g}$, we have a negative contribution to the left-hand side of (4.8). In addition, in some polymer-penetrant systems, $\left[C_{x}\right]_{s}<0$. Therefore, we conclude that in certain polymer-penetrant systems $a$ will be negative.

In order to make the problem analytically tractable, we make one more simplifying assumption. As stated before, the diffusion coefficient often, though not always, increases dramatically as the polymer goes from the glassy to rubbery state. However, changes within phases are less important. Hence, we perform the same averaging as we did with the relaxation time to obtain the following form for $D(C)$ :

$$
D(C)= \begin{cases}D_{g}, & 0 \leq C \leq C_{*}, \\ D_{r}, & C>C_{*} .\end{cases}
$$

Since we have chosen this simplistic form, equation (4.7) may be written

$$
C_{t}=D(C) C_{x x}+E \sigma_{x x} .
$$


Taking advantage of the fact that all our functions are now piecewise constant, we may combine equations (4.10) and (4.2) to yield the following equation for $C$ (which also holds for $\sigma$ ):

$$
C_{t t}=[D(C)+\nu E] C_{x x t}-\beta(C) C_{t}+[\beta(C) D(C)+\mu E] C_{x x} .
$$

Note that the classical technique of seeking similarity solutions will not in general solve an equation of the form of (4.8) or (4.11). Boley [21] developed an integralequation solution technique to solve some classes of moving-boundary problems for which no similarity solutions exist. We have used this technique extensively and with great success. To begin, we introduce two new functions $T^{g}$ and $T^{r}$ which are the extensions of $C^{g}$ and $C^{r}$ to the entire domain and which satisfy the following equations:

$$
\begin{gathered}
T_{t t}^{g}=\left(D_{g}+\nu E\right) T_{x x t}^{g}-\beta_{g} T_{t}^{g}+\left(\beta_{g} D_{g}+\mu E\right) T_{x x}^{g}, \quad x>0, \\
T^{g}(x, t)=C^{g}(x, t), \quad x>s(t),
\end{gathered}
$$

$$
T^{g}(x, 0)=C_{i}(x)
$$

$$
T^{g}(0, t)=g_{b}(t)
$$

$$
T^{g}(s(t), t)=C_{*}, \quad T_{t}^{g}(s(t), t)>0,
$$

$$
T_{t t}^{r}=\left(D_{r}+\nu E\right) T_{x x t}^{r}-\beta_{r} T_{t}^{r}+\left(\beta_{r} D_{r}+\mu E\right) T_{x x}^{r}, \quad x>0
$$

$$
T^{r}(x, t)=C^{r}(x, t), \quad 0<x<s(t)
$$

$$
T^{r}(x, 0)=g_{i}(x)
$$

$$
T^{r}(0, t)=C_{b}(t)
$$

$$
T^{r}(s(t), t)=C_{*}, \quad T_{t}^{g}(s(t), t)>0,
$$

The functions $g_{b}(t)$ and $g_{i}(x)$ are necessary since we have extended equations (4.12a) and (4.15a) outside their region of validity. Since equations (3.3) only hold in the domain of validity of (3.1), we must introduce these fictitious boundary conditions. The systems of equations (4.12)-(4.13) and (4.15)-(4.16) can be solved using standard Green's function techniques. The resulting solutions, which are written as convolutions of $g_{b}(t)$ and $g_{i}(x)$, can then be substituted into equations (4.14), (4.17), and (4.18) to 
yield a system of three integral equations for the three unknowns $g_{b}(t), g_{i}(x)$, and $s(t)$. Asymptotic and numerical methods can then be employed to solve these equations.

Though we are now using linear operators, note that we have introduced nonlinearities in $\beta(C)$ and in (4.18), which still contains $\sigma$, which is a decidedly nonlinear function of $C$. Therefore, in certain parameter ranges one must contend with notions of nonuniqueness and bifurcation [22]. In this paper we simply wish to outline the solution process; a brief result is presented in the next section.

5. Analytical results for a dissolution problem. We now model a polymer entanglement network dissolving in the presence of a solvent, the concentration of which will be denoted by $C$. To demonstrate the unusual behavior encountered, what follows is a sketch of our solution technique; an expanded discussion of our solution techniques, as well as solutions to other polymer-penetrant system models, appears in [22]-[24]. In this particular experiment, any change in diffusion coefficient does not affect the physics, so we may take $D_{g}=D_{r}=D$. We begin by nondimensionalizing our problem in the following manner:

$$
\text { length scale: } \sqrt{\frac{D}{\beta_{r}}}, \quad \text { time scale: } \frac{1}{\beta_{r}}, \quad \frac{\beta_{g}}{\beta_{r}}=\epsilon, \quad 0<\epsilon \ll 1 \text {. }
$$

From experimental observations, we also expect the stress contribution to be important, so we let $\mu=\mu_{0} \epsilon^{-2}$. We hypothesize that the following expansions in $\epsilon$ hold:

$$
\begin{gathered}
T^{g}=T^{0 g}+o(1), \quad T^{r}=T^{0 r}+o(1), \\
\sigma^{g}=\epsilon^{-1} \sigma^{0 g}+o\left(\epsilon^{-1}\right), \quad \sigma^{r}=\epsilon^{-1} \sigma^{0 r}+o\left(\epsilon^{-1}\right) .
\end{gathered}
$$

Now substituting equations (5.1) and (5.2) and our expressions for $D$ and $\mu$ into equations (4.12a), (4.2), (4.15a), and (4.18), we have (to leading orders)

$$
\begin{gathered}
T_{t t}^{0 g}=\kappa^{2} T_{x x}^{0 g}, \\
\sigma_{t}^{0 g}=\frac{\kappa^{2}}{\gamma} T^{0 g}, \quad x>s(t), \\
T_{t t}^{0 r}=\kappa^{2} T_{x x}^{0 r}-T_{t}^{0 r}, \\
\alpha \epsilon\left[T_{x}^{0}\right]_{s}-\gamma \frac{\sigma^{0 g}(s(t), t)}{\dot{s}}=a \dot{s},
\end{gathered}
$$

where $\kappa^{2}=\mu_{0} E / \beta_{g} D, \gamma=\nu E / D$, and $\alpha=1+\gamma$. In a standard Stefan problem, the same operator hold on both sides of the front, but the coefficients are different. Note that here the problem is much more complicated; here two different operators hold on each side of the front. This extra complication also makes our system (5.3)-(5.5) worthy of further theoretical study for existence and uniqueness of solutions.

It is clear that in this problem if $\left[T_{x}^{0}\right]=O(1)$, then $a<0$, since we expect $\sigma>0$ and $\dot{s}>0$. Even if $\left[T_{x}^{0}\right]>O(1), a$ may still be negative if the concentration at the front exhibits a nonstandard profile. This confirms our earlier suspicions that the 
parameter $a$ cannot be given a simple physical interpretation. However, for reasons that will become clear later we wish to restrict $a$ to the following range:

$$
a<0, \quad C_{*} \leq|a| \leq 1 .
$$

In this problem, the polymer is dry when the experiment starts, so equation (4.13a) becomes

$$
T^{g}(x, 0)=0 .
$$

We now wish to model a polymer entanglement network dissolving in the presence of a solvent. Here $C$ is the concentration of the solvent. Imagine an experiment in which a polymer matrix is exposed to a infinite well of diluent. Though the concentration of the diluent may be 1 at the edge of the polymer matrix, it is clear that at the instant that we introduce the polymer into the solvent, the concentration can be no greater than $C_{*}$, which is now defined as that concentration at which the entanglement network dissolves. We would expect that the maximal concentration of the diluent at the boundary will be achieved only in the mathematical limit $t \rightarrow \infty$. This motivates our boundary condition

$$
C\left(0^{+}, t\right)=C_{b}(t)=1-\left(1-C_{*}\right) e^{-r t}, \quad r \neq 1,
$$

where $r$ is a constant. We have included the requirement $r \neq 1$ to eliminate the presence of logarithmic singularities in our solutions.

For $x>\kappa t$ the solution to our outer problem is exactly 0 . We will call the line $x=\kappa t$ the primary front, referring to the fact that it is the first signal to reach a certain point. However, it is not the moving boundary between the two phases at which (4.18) holds. That boundary is the secondary front $x=s(t)$, which we define to be the curve where the network is completely dissolved. In other words, it is where both conditions in (4.14) and (4.17) hold. It is clear that $s(t)<\kappa t$ for all $t$. We see from equation (5.3a) that the characteristics of the outer problem carry some constant value $T^{0 g}=C_{1}$ forward with speed $\kappa$, so there must exist a "mushy region" $s(t)<x<\kappa t$ where $T^{0 g} \equiv C_{1}$. It can be shown that there can be no other boundary layer in the glassy polymer, so $C_{1}=C_{*}$. This is illustrated in Fig. 1.

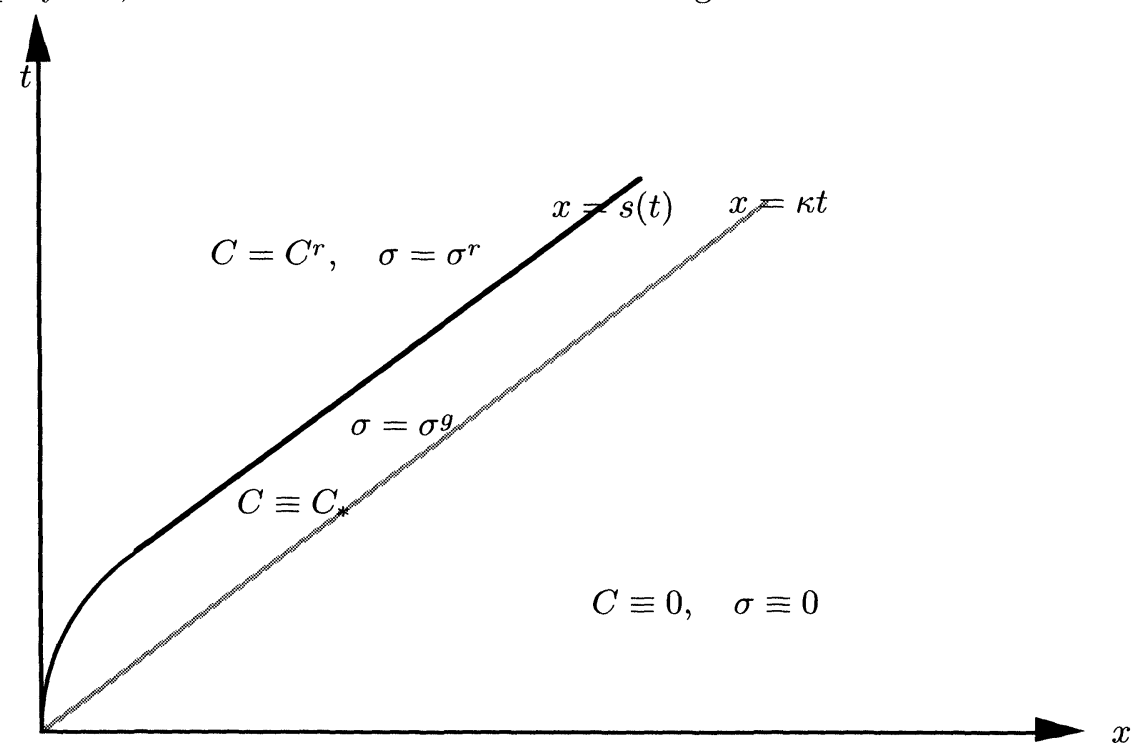

FIG. 1. Regions of validity for different outer representations. 
Since our full equation cannot support the discontinuity found in the solution of the outer problem, we must construct a boundary layer around the subcharacteristic $x=\kappa t$, giving us the uniformly valid solution in the glassy region

$$
T^{u g}(x, t)=\frac{C_{*}}{2} \operatorname{erfc}\left(\frac{x-\kappa t}{\sqrt{2 \alpha \epsilon t}}\right) .
$$

Equation (5.9) is the standard functional form that appears when a smoothing term is eliminated in an outer expansion and a hyperbolic equation results.

Since (4.11) [and hence (5.3a)] also hold for $\sigma$, we see that $\sigma \equiv 0$ for $x>\kappa t$. Therefore, in the mushy region, we may use this fact and immediately solve $(5.3 \mathrm{~b})$ to yield

$$
\sigma^{0 g}(x, t)=\frac{\kappa^{2} C_{*}}{\gamma}\left(t-\frac{x}{\kappa}\right)
$$

Hence, in order for $\sigma^{0 g}$ stay bounded (which is what we expect on physical grounds), we see that

$$
s(t) \sim \kappa t-s_{\infty}+s_{1}(t) \quad \text { as } t \rightarrow \infty,
$$

where $s_{\infty}>0$ and $s_{1}(t) \rightarrow 0$ as $t \rightarrow \infty$. Using equation (5.10) evaluated at our secondary front, we see that equation (5.5) becomes

$$
\alpha \epsilon\left[C_{x}\right]_{s}-\frac{\kappa^{2} C_{*}}{\dot{s}}\left[t-\frac{s(t)}{\kappa}\right]=a \dot{s} .
$$

It can be shown that a boundary layer exists in the rubbery region, and that the uniformly valid solution is given by

$$
T^{u r}(x, t)=T^{0 r}(x, t)+\left[C_{*}-T^{0 r}(s(t), t)\right] \exp \left\{\frac{\left(\kappa^{2}-\dot{s}^{2}\right)[x-s(t)]}{\alpha \epsilon \dot{s}}\right\} .
$$

Using (5.13) in (5.12), we have

$$
\left[T^{0 r}(s(t), t)-C_{*}\right]\left(\kappa^{2}-\dot{s}^{2}\right)-\kappa^{2} C_{*}\left[t-\frac{s(t)}{\kappa}\right]=a \dot{s}^{2} .
$$

Using the integral method, we can solve equations (4.15), (4.16a), and (5.8) to yield

$$
\begin{gathered}
T^{0 r}(x, t)=g_{i}(x)+\kappa \int_{0}^{t}\left[g_{i}^{\prime}(x+\kappa z)-g_{i}^{\prime}(|x-\kappa z|)\right] g_{k}(z, t) d z-2 g_{i}(0) g_{k}(x / \kappa, t) \\
+\frac{x}{2} \int_{x / \kappa}^{t} e^{-z / 2}\left[1-\left(1-C_{*}\right) e^{-r(t-z)}\right] \frac{I_{1}\left(\sqrt{\kappa^{2} z^{2}-x^{2}} / 2 \kappa\right)}{\sqrt{\kappa^{2} z^{2}-x^{2}}} d z \\
+\left[1-\left(1-C_{*}\right) e^{-r(t-x / \kappa)}\right] e^{-x / 2 \kappa}
\end{gathered}
$$

where

$$
g_{k}(z, t)=\left[\frac{e^{-z / 2}}{2}+\frac{z}{4} \int_{z}^{t} e^{-y / 2} \frac{I_{1}\left(\sqrt{y^{2}-z^{2}} / 2\right)}{\sqrt{y^{2}-z^{2}}} d y\right] H(t-z)
$$


Performing long-time asymptotics on equations (5.15) and substituting our results in (4.17) and (5.14), we obtain the results that

$$
s_{\infty}=\frac{|a| \kappa}{C_{*}}, \quad T^{0 r}(s(t), t) \sim|a| .
$$

Then we see from $C_{*} \leq C^{0 r} \leq 1$ that we have our compatibility condition (5.6).

We next perform short-time asymptotics on equations (5.15). Substituting our results into equations (4.17) and (5.14), we have an interesting result: in order for our solutions to be twice continuously differentiable, we must have that

$$
r=\frac{C_{*}}{1-C_{*}} .
$$

What does this mathematical constraint mean physically? It says that in order for our dissolution front to propagate, the concentration at the interface between the polymer and the reservoir must be regulated by the polymer network itself. Thus $r$ in some sense represents the internal dissolution rate of the polymer and could be related to the strength of the entanglement network.

Going to the next order in the asymptotics yields the result that

$$
s(t) \sim \frac{\kappa C_{*} t^{2}}{2 \sqrt{3|a|\left(1-C_{*}\right)}} .
$$

Figure 2 shows a plot of our superimposed asymptotic expansions for the listed set of parameter values. The grey line is the primary front. The narrow lines are the graphs of our actual asymptotic expansions (5.16) and (5.18), while the thicker line is simply a sketch of the way the actual front would interpolate between these two expansions. Note that there are several important results here from an experimental point of view. By simply performing the experiment heretofore outlined, one can determine $\kappa$ (from the front speed), $C_{*}$ (from the concentration in the mushy region), and $a$ (from the width of the mushy region). In fact, Fig. 2 accurately captures the qualitative behavior of a polymer such as poly-methyl-methocrylate dissolving in the presence of a solvent such as toluene [25].

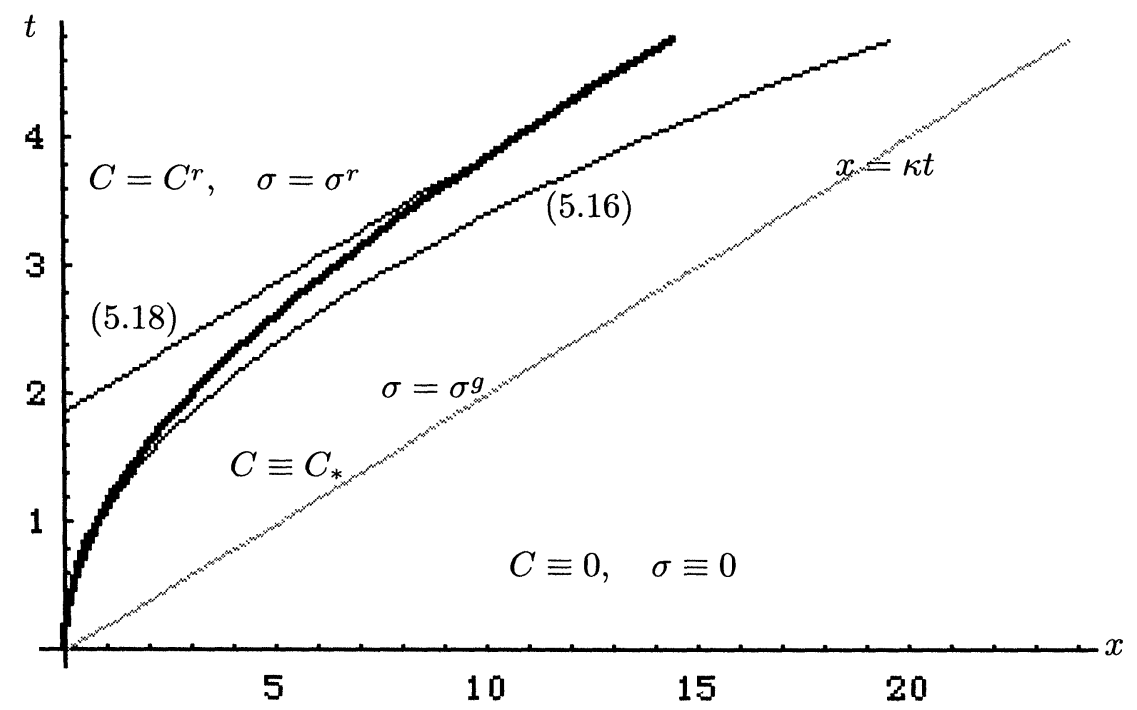

FIG. 2. Superimposed asymptotic front expansions, $C_{*}=0.4, a=-0.75, \kappa=5$. 
Though in this section we concerned ourselves primarily with calculating the motion of the front, in [22]-[24] we supply the details of our calculations, which include asymptotic estimates of the stress and concentration profiles.

6. Conclusions. New materials and their large number of widely varied applications have revolutionized several scientific fields. This has led the engineering community to clamor for coherent mathematical models in order better to control the design of such materials. The standard Fickian diffusion model is insufficient to explain the phenomena observed, including sharp fronts moving with constant speed and fronts where the concentration flux behind the front is less than that ahead of the front.

By simplifying our general model (1.1), we are able to model the most salient nonstandard feature of many classes of polymer-penetrant systems: a nonlocal "memory" effect which induces a viscoelastic stress. This effect varies between the polymer phases, as does the diffusion coefficient. The moving boundary-value problem which ensues has not received much attention in the mathematical literature. Further study is needed of this boundary condition to study the existence and uniqueness of solutions to such a condition, especially since this condition is not solvable by similarity solutions.

We rely upon an integral method developed by Boley [21] which gives solutions which are not in closed form. By using a perturbation expansion in a suitable small parameter, we were able to reduce the problem to one where two different operators held on either side of the front. The nonlinearities and complications inherent in such a problem merit further study, especially in the area of bifurcation analysis.

However, in the case we present, we obtain asymptotic estimates for the motion of the front. In [22]-[24] we demonstrate the details of these calculations and how they are used to obtain the concentration and stress profiles for the problem treated here and for several additional problems arising in other applications.

\section{Nomenclature.}

\subsection{Variables and parameters.}

$a$ : coefficient in flux-front speed relationship (2.3).

A: matching constant.

$C(\mathbf{x}, t)$ : concentration of penetrant or diluent at position $\mathbf{x}$ and time $t$.

$D(C)$ : binary diffusion coefficient for system.

$E(C)$ : coefficient preceding the stress term in the modified flux equation (2.4a).

$f\left(C, C_{t}\right)$ : arbitrary function in viscoelastic stress term.

$\mathcal{F}_{n}[C]$ : nonlinear differential operator on $C$.

$g(\cdot)$ : arbitrary function, variously defined.

$\mathcal{G}_{n}[\cdot]$ : hereditary kernel.

$H(\cdot)$ : Heaviside step function, defined as 0 for negative argument and 1 for positive argument.

$I_{n}(\cdot)$ : the $n$th modified Bessel function.

$\mathbf{J}(\mathbf{x}, t)$ : flux at position $\mathbf{x}$ and time $t$.

$n$ : indexing integer.

$r$ : dimensionless parameter in dissolution problem boundary condition (5.8).

$\mathbf{s}(t)$ : position of secondary front, defined as $C(\mathbf{s}(t), t)=C_{*}$ and $C_{t}(\mathbf{s}(t), t)>0$.

$\bar{s}(x)$ : the inverse function of $x=s(t)$, written as $t=\bar{s}(x)$.

$t$ : time from imposition of external concentration.

$T(x, t)$ : imbedding of $C$ from one region to the fully semi-infinite region. 
$x$ : one-dimensional spatial variable.

$\mathbf{x}$ : three-dimensional spatial variable.

$y$ : dummy integration variable.

$z$ : dummy integration variable.

$\mathcal{Z}$ : the integers.

$\alpha$ : nondimensional parameter, value $1+\gamma$.

$\beta(C)$ : inverse of the relaxation time.

$\gamma$ : nondimensional parameter, value $\nu E / D$.

$\delta_{1 n}:$ the Kronecker delta function.

$\delta(\cdot)$ : the Dirac delta function.

$\epsilon$ : perturbation expansion parameter, value $\beta_{g} / \beta_{r}$.

$\kappa$ : nondimensional parameter, value $\sqrt{\mu_{0} E / \beta_{g} D}$.

$\mu$ : coefficient of concentration in stress evolution equation (4.2).

$\nu$ : coefficient of $C_{t}$ in stress evolution equation (4.2).

$\sigma(\mathbf{x}, t)$ : stress in polymer at position $\mathbf{x}$ and time $t$.

$\Omega$ : region occupied by the polymer.

$\partial \Omega$ : boundary of the region $\Omega$.

\subsection{Additional notation.}

$b$ : as a subscript, used to indicate a quantity at the boundary of the polymer.

$g$ : as a sub- or superscript, used to indicate the glassy state.

$i$ : as a subscript, used to indicate an quantity at $t=0$.

$n \in \mathcal{Z}$ : as a superscript, used to indicate a term in an expansion in $\epsilon$; as a subscript, used to indicate a particular region of the polymer or a term in an expansion in $t$.

$k$ : as a subscript, used to indicate a kernel.

$r$ : as a sub- or superscript, used to indicate the rubbery state.

$t$ : as a subscript, used to indicate partial differentiation with respect to $t$.

$u$ : as a superscript, used to indicate a uniform expansion.

$x$ : as a subscript, used to indicate partial differentiation with respect to $x$.

': with a function, used to indicate differentiation with respect to $x$; with an independent variable, used to indicate a dummy integration variable.

$\because$ used to indicate differentiation with respect to $t$.

*: as a subscript, used to indicate a matching value between two states or two representations.

$\infty$ : as subscript, used to indicate a term in an expansion in $t$.

$[\cdot]_{\mathbf{s}}:$ jump across the front $\mathbf{s}$, defined as $\cdot g\left(\mathbf{s}^{+}(t), t\right)-\cdot r\left(\mathbf{s}^{-}(t), t\right)$.

Acknowledgments. The authors wish to thank Thomas Witelski and Christopher Durning for their contributions, both direct and indirect, to this paper. Many of the calculations herein were performed using Maple.

\section{REFERENCES}

[1] E. Martuscelli and C. Marchetta, eds., New polymeric materials: reactive processing and physical properties, Proceedings of International Seminar, 9-13 June 1987, Naples, Italy. VNU Science Press, Utrecht, the Netherlands, 1987.

[2] S. R. Shimabukuro, Stress Assisted Diffusion in Polymers, Ph.D. thesis, California Institute of Technology, 1990.

[3] P. J. TARche, Polymers for Controlled Drug Deliveries, CRC Press, New York, 1991. 
[4] T. J. Roseman and S. Z. Mansdorf, eds. Controlled Release Delivery Systems, Marcel Dekker, New York, 1983.

[5] R. LANGer, New methods of drug deliveries, Science, 249 (1990), pp. 1527-1534.

[6] L. F. Thompson, C. G. Wilson, And M. J. Bowden, Introduction to Microlithography, ACS Symposium Series, vol. 219, ACS, Washington, D.C., 1983.

[7] J. S. Vrentas, C. M. Jorzelski, And J. L. Duda, A Deborah number for diffusion in polymersolvent systems, AIChE J., 21 (1975), pp. 894--901.

[8] N. Thomas and A. H. Windle, A theory of Case II diffusion, Polymer, (1982), pp. 529-542.

[9] J. CRAnK, Free and Moving Boundary Problems, Oxford University Press, New York, 1984.

[10] D. S. Cohen And A. B. White, JR., Sharp fronts due to diffusion and viscoelastic relaxation in polymers, SIAM J. Appl. Math., 51 (1991), pp. 472-483.

[11] - Sharp fronts due to diffusion and stress at the glass transition in polymers, Los Alamos Technical Report 88-2081, June 1988; J. Polymer Sci., Part B: Polymer Physics, 27 (1989), pp. 1731-1747.

[12] C. K. HAYES AND D. S. Cohen, The evolution of steep fronts in non-Fickian polymer-penetrant systems, J. Polymer Sci., Part B: Polymer Physics, 30 (1992), pp. 145-161.

[13] R. W. Cox AND D. S. CoHEn, A mathematical model for stress-driven diffusion in polymers, J. Polymer Science B: Polymer Physics, 27 (1989), pp. 589-602.

[14] R. W. Cox, A Model for Stress-driven Diffusion in Polymers, Ph.D. thesis, California Institute of Technology, 1988.

[15] C. K. Hayes, Diffusion and Stress Driven Flow in Polymers, Ph.D. thesis, California Institute of Technology, 1990.

[16] D. S. Cohen, A. B. White, Jr., And T. P. Witelski, Shock Formation in a Viscoelastic Diffusive System, SIAM J. Appl. Math., 55 (1995), pp. 348-368.

[17] J. Crank, The Mathematics of Diffusion, 2nd ed., Clarendon Press, Oxford, 1976.

[18] N. Thomas And A. H. Windle, Transport of methanol in poly-(methyl-methocry-late), Polymer, 19 (1978), pp. 255-265.

[19] C. J. Durning, Differential sorption in viscoelastic-fluids, J. Polymer Sci., Polymer Phys. Ed., 23 (1985), pp. 1831-1855.

[20] W. G. KNAUSS AND V. H. Kenner, On the hygrothermomechanical characterization of polyvinyl acetate, J. Appl. Phys., 51 (1980), pp. 5131-5136.

[21] B. A. BOLEY, A method of heat conduction analysis of melting and solidification problems, J. Math. Phys, 40 (1961), pp. 300-313.

[22] D. A. Edwards, Constant front speed in weakly diffusive non-Fickian systems, SIAM J. Appl. Math, 55 (1995), to appear.

[23] D. A. Edwards And D. S. Cohen, A mathematical model of a dissolving polymer, AIChE. J., submitted.

[24] D. A. EDWARDS, The effect of a varying diffusion coefficient in polymer-penetrant systems, IMA J. Appl. Math., submitted.

[25] J. C. Wu, Quantum Chemical Process Research Center, private communication, March 11, 1994. 